\title{
White-tailed spider bite over the posterior ankle causing significant reaction in the groin: Presenting in the UK
}

\author{
Onyebuchi-iwudibia $\mathrm{O}^{1 *}$ and Banks $\mathrm{LN}^{2}$ \\ ${ }^{1}$ GP Registrar, Peterborough City Hospital, UK \\ ${ }^{2}$ Consultant Trauma and Orthopaedics, Glan Clwyd Hospital, UK
}

\begin{abstract}
Background: White-tail spiders are considered poisonous. However, the spectrum of symptoms attributed to their bites is of great interest and has resulted in widely conflicting literature.

Case summary: A 60 year old lady who was bitten by a white-tail spider in Australia presented to a general district hospital in North Wales with symptoms of right groin and thigh pain, thigh swelling and overlying cellulitis. C-reactive protein white blood cell count and D-dimer serum blood tests were all raised on presentation to the hospital. A doppler ultra-sound scan ruled out a deep vein thrombosis, whilst a magnetic resonance scan showed subcutaneous edema and fluid collection in the regions of the groin The patient was treated with intravenous antibiotics and her symptoms resolved. No recurrence was seen at follow-up appointment.
\end{abstract}

Discussion: The case supports literature attributing systemic symptoms to white-tail spider bites as opposed to those suggesting that such diagnoses are inappropriate. However, multidisciplinary approach should be advocated when dealing with suspected spider bites to ensure other possible diagnoses are ruled out.

\section{Introduction}

Australian white-tail spiders (Lampona cylindrata and L. murina) are considered to be among the poisonous Australian spiders [1,2]. They are so-called 'white-tail' because of the whitish tail at the end of their abdomen (Figure 1) [3].

The spectrum of symptoms caused by these spiders is a subject of great interest with conflicting literature. Some of the literature suggests that bites from these spiders are responsible for skin blistering, ulceration and necrosis collectively termed 'necrotising arachnidism' $[1,4]$, whereas other articles suggest that the diagnoses in many cases are inappropriate and if patients are properly investigated, alternative and more appropriate diagnoses will be made [5-7]. One group [6] examined cases of necrotising arachnidism and found that there is little or no evidence to suggest that there was a definite spider bite and in most cases, alternative diagnoses were made following thorough investigation. The former school of thought, however, attributes necrotizing dermatitis to white-tail spider bite with a recurrence of symptoms in many cases [1]. Many other systemic effects of white-tail spider bites have been reported. This conflicting literature with little or no consensus on the signs and symptoms of white-tail spider bites, can lead to a delay in diagnosis in suspected cases with a median time to diagnosis reported as three weeks [8]. It is however, not widely disputed that spider bites are responsible for localized symptoms of swelling, redness and pain $[8,9]$.

Although there are a number of case studies of Australian whitetail spider bites in Australia and New Zealand, there is a paucity of such case studies in the UK.

\section{Clinical case}

A 60-year-old female was referred to the Orthopaedic team in a busy district General Hospital in North Wales complaining of a painful and swollen right groin and thigh.

She informed the department that she had been bitten by a whitetail spider (Figure 1) in Australia around the posterior aspect of her ankle, about 7 days previously. She had received treatment with the local pharmacist (after describing the spider to the pharmacist) at the time and had then flown to back to the United Kingdom 5 days later. She had no other past medical or family history of note.

On examination, there was a fluctuant, tender mass (approximately $7 \times 5 \times 3 \mathrm{~cm}$ ) on the medial aspect of the right thigh, which was surrounded by cellulitis. A healing blister was seen on the posterior aspect of her ankle overlying the tendo-achilles. She remained afebrile.

Her serum blood tests showed an elevated C-reactive protein $(30.3 \mathrm{mg} / \mathrm{L}[<5.0 \mathrm{mg} / \mathrm{L}])$, white cell count $\left(13.6 \times 10^{\wedge} 9 / \mathrm{L}[4.0-11.0 \times\right.$

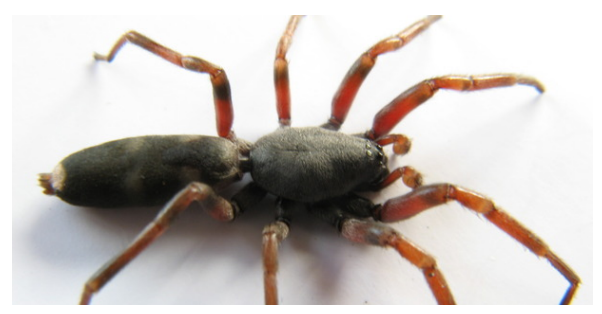

Figure 1. Photograph of a white-tailed spider (native of southern and eastern Australia).

Correspondence to: Oscar Onyebuchi-iwudibia [MBBS, MSc], GP Registrar, Peterborough City Hospital, Edith Cavell Campus, Peterborough, PE3 9GZ, E-mail: oscariwudibia@yahoo.co.uk

Key words: white-tail spider, groin pain, cellulitis, lymphadenopathy

Received: July 21, 2016; Accepted: August 09, 2016; Published: August 12, 2016 


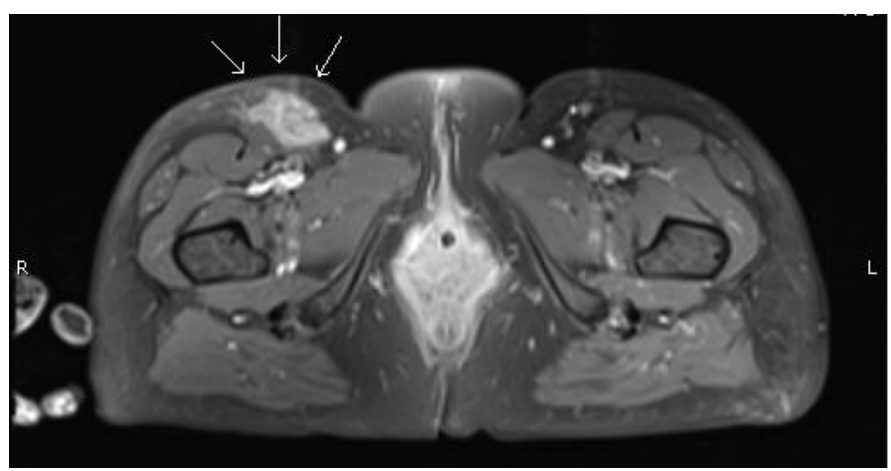

Figure 2. Axial MRI of the pelvis and proximal thigh. Arrows indicate area of oedema presenting as large soft tissue masses.

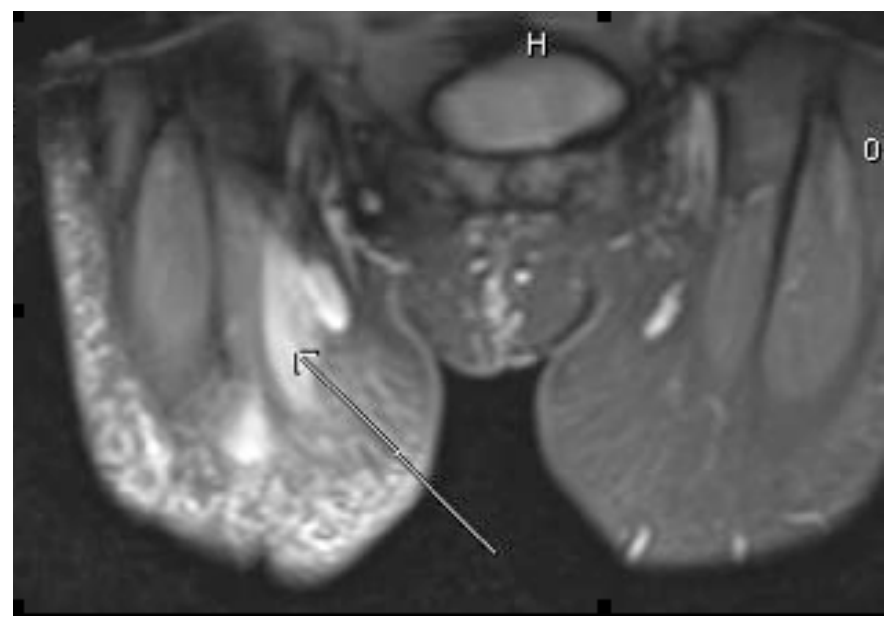

Figure 3. Coronal MRI of the pelvis and proximal thigh. Arrows indicate area of oedema presenting as large soft tissue masses.

109/L]) and D-dimer (1255 ng/ml [<500 ng/ml]). Blood cultures were negative. The patient was started on intravenous (IV) antibiotics for the cellulitis (Clindamycin $600 \mathrm{mg}$ four times a day, given that the patient was penicillin allergic). After 24 hours and little clinical improvement, the antibiotic regimen was changed to Meropenem ( $1 \mathrm{~g}$ three times per day) after discussion with the local microbiology team.

A doppler ultrasound of the right lower limb ruled out a deep vein thrombosis (DVT). A plain pelvis radiograph and review by the general surgical team did not reveal any intra-abdominal abnormalities. After 24 hours of IV antibiotics, several more soft tissue, tender masses were noticed around the patient's inguinal region. A magnetic resonance scan (MRI) of the pelvis and thigh was therefore obtained on day 4 of admission (Figures 2 and 3) showing; "evidence of superficial fluid accumulation extending from the right inguinal area medially into the upper, superficial aspect of the right thigh. The changes were consistent with subcutaneous oedema and active cellulitis". There was a concern that the "more localized fluid accumulation in the right groin area could represent abscess formation" and ultrasonic follow up was recommended if the soft tissue masses did not dissipate.

The National Poison Information Services and local infectious diseases unit were both contacted for further advice in managing this patient. Both centres had very little experience with Australian insect bites and couldn't offer any further management advice given the paucity of patients presenting with such problems in the United Kingdom (UK).
The patient's soft tissue masses and cellulitis settled over 6 days and she was discharged with a follow-up clinic appointment the following week. At follow-up she was completely asymptomatic with no recurrence of any symptoms. There has been no further recurrence of symptoms in the following three months.

\section{Discussion}

The case study illustrates that white-tail spider bites are capable of causing systemic symptoms that require prompt treatment. This lends credence to literature suggesting that white-tail spider bites cause a spectrum of systemic symptoms as opposed to localized symptoms. In this case, the patient identified that a white-tail spider had bitten her and visited a local pharmacist immediately where anti-inflammatory agents were administered.

Also, although there was a clear history of Australian spider bite, the managing Orthopaedic team was obliged to thoroughly investigate the patient to rule out other diagnoses. Although the patient did not develop the classical 'necrotising arachnidism' attributed to whitetail spider bites $[1,4]$, the case clearly illustrates that these spider bites cause a range of systemic symptoms which can be confused with other diagnosis such as DVT (amongst others) in this case, considering the constellation of long haul flight, painful, swollen thigh and markedly raised D-dimer. This case contradicts literature suggesting that these spider bites are usually not responsible for severe systemic symptoms [8]. It does however provide support to the literature suggesting systemic symptoms of white-tail spider bites, but not as to the history of recurrence [1]. It is possible that it is too early to assess recurrence but it is unlikely. This case adds to the theories that the effects of white-tail spider bites are varied and the factors responsible for these variations could constitute a subject for research.

In conclusion, although there is literature to suggest that the wide array of signs and symptoms attributed to white-tail spider bites may be inappropriate, a high index of suspicion is essential when dealing with suspected cases of spider bites. Such cases should therefore be thoroughly investigated to rule out other diagnoses. A multidisciplinary approach would be paramount in these cases. Following appropriate diagnosis, we feel publication of case reports would be helpful in creating a body of evidence, especially for physicians in the UK with regards to the presentations of spider bites and the responses to various treatment modalities.

\section{References}

1. Pincus SJ, Winkel KD, Hawdon GM, Sutherland SK (1999) Acute and recurrent skin ulceration after spider bite. Med J Aust 171: 99-102. [Crossref]

2. Ní Chróinín D, Cummins F, O'Connor P (2009) Australian immigrant of the arachnid variety? Eur J Emerg Med 16: 159-162. [Crossref]

3. Australian Museum. White-tailed spider2014 29th April 2014. Available from: http:// australianmuseum.net.au/White-tailed-Spider.

4. Spring WJ (1987) A probable case of necrotizing arachnidism. Med J Aust 147: 605 607. [Crossref]

5. White J (1999) Necrotising arachnidism. Med J Aust 171: 98. [Crossref]

6. Isbister GK, Gray MR (2003) White-tail spider bite: a prospective study of 130 definite bites by Lampona species. Med J Aust 179: 199-202. [Crossref]

7. Banks J, Sirvid P, Vink C (2004) White-tailed spider bites--arachnophobic fallout? NZ Med J 117: U748. [Crossref] 
Onyebuchi-iwudibia O (2016) White-tailed spider bite over the posterior ankle causing significant reaction in the groin: Presenting in the UK

8. Isbister GK, Whyte IM (2004) Suspected white-tail spider bite and necrotic ulcers. Intern Med J 34: 38-44. [Crossref]
9. Nentwig W, Gnädinger M, Fuchs J, Ceschi A (2013) A two year study of verified spider bites in Switzerland and a review of the European spider bite literature. Toxicon 73 104-110. [Crossref]

Copyright: (C2016 Onyebuchi-iwudibia O. This is an open-access article distributed under the terms of the Creative Commons Attribution License, which permits unrestricted use, distribution, and reproduction in any medium, provided the original author and source are credited. 